\title{
Cosmic Topologies of Imitation: From the Horror of Digital Autotoxicus to the Auto-Toxicity of the Social
}

\section{Paper accepted June $15^{\text {th }} \mathbf{2 0 1 6}$ for special issue of Parallax on Autoimmunities - Please refer to published version due early 2017.}

\author{
Tony D. Sampson (University of East London)
}

This article expands on an earlier concept of horror autotoxicus linked to digital contagions of spam and network Virality. $^{1}$ It aims to present, as such, a broader conception of cosmic topologies of imitation (CTI) intended to better grasp the relatively new practices of social media marketing. Similar to digital autotoxicity, CTI provide the perfect medium for sharing while also spreading contagions that can potentially contaminate the medium itself. However, whereas digital contagions are perhaps limited to the toxicity of a technical layer of information viruses, the contagions of CTI are an all pervasive auto-toxicity which can infect human bodies and technologies increasingly in concert with each other. This is an exceptional autotoxicus that significantly blurs the immunological line of exemption between self and nonself, and potentially, the anthropomorphic distinction between individual self and collective others.

This earlier work on digital culture discussed the significant role of contagion in virus writing cultures and online communication practices, where, in short, the tendency for codes and communication messages to spread like viruses on a network inspired an extremely profitable anti-virus industry and nascent viral marketing business. On one hand, biological analogies ushered in an anti-virus discursive formation that determined, to some extent, what people can and cannot do on a network by distinguishing between good and bad digital code in a similar way to which organic immune systems are assumed to exempt the threat posed by anomalous nonself cells from those of the self. On the other hand though, prediscursive forces were identified in the social spreading of biologically derived anxieties linked to these appeals to immunity and efforts made to trigger affective contagions associated with viral marketing practices. Indeed, in many ways the concept of horror autotoxicus was initially introduced as a way to explain how immunologic inspired digital systems become vulnerable to viral communication environments in which contagious anomalies are constituent rather than exempt. In this context, autoimmunity is a useful concept because it challenges the immunologic principles of the self/nonself binary relation and helps to identify discursive formations and prediscursive forces that arrange social relations by way of contamination rather than immunity. Indeed, taken forward to an all-pervasive corporate social media era in which a second wave of viral marketing has arguably come of age, this article revisits horror autotoxicus to argue that the virality of digital culture can now be grasped through a prevailing auto-toxicity of the social.

In a very concrete fashion the now infamous Facebook research on emotional contagion in 2014 (discussed below) evidences how a continued focus on contagion theory adds to a much needed critical study of digital culture. The rise of corporate social media in the last decade has indeed necessitated a reenergizing of these critical approaches. As follows, 
Langlois and Elmer distinguish between three different yet intertwined approaches that are significant to this discussion. ${ }^{2}$ Firstly, the virality of social media is closely related to a critical economy approach that understands corporate endeavours to mine social life through appeals to such things as emotional, felt and affective user experiences with software. Social media does more than merely capture the attention of users and sell it on to marketers. It produces user performances that marketers can readily mine, manipulate and nudge into action. Secondly, the conceptual work developed in this article is directly linked to empirical work focused on the algorithms that function behind the scenes on social media platforms. The conceptualization of CTI therefore recognizes how capricious affective contagions are nudged into action by way of analytics triggered by, for example, a click on a like or share button. Lastly, by helping to illuminate the manipulation of affective user experiences at the interface of corporate social media this discussion feeds into a third software activist approach to corporate social media with a view to awakening 'new user agencies' ${ }^{3}$ beyond those social relations already configured by corporate marketers.

\section{Rethinking Immunity through Autoimmunity}

Autoimmunity is a concept that explores beyond earlier territorializations of immunological exemption to ever more complex stratagems that tap into contagious social arrangements in digital culture. Yet, it is important to begin by noting that autoimmunity is not opposed to immunologic. To grasp its full purchase we need to approach autoimmunity by thinking through immunology. As set out in this special issue, autoimmunity is conventionally studied within the field of immunology. The concept refers to an immunologic phenomenon whereby an organism mounts an immune response against its own tissues; a paradoxical situation in which self-defence (immunity, protection) manifests as self-harm (pathology). Today, the term autoimmunity is used to account for any instance in which the body fails to recognise its own constituents as 'self', an error that results in self-harm or injury.

Autoimmunity is however regarded as a controversial concept within immunology. It is important, as such, to grasp the flaws in Paul Ehrlich's original concept of horror autotoxicus. To begin with, in crude terms autoimmunity is posited as an impossibility because all organisms are so horrified by self-discrimination that they will selectively avoid self-toxicity. The later discovery of the harsh realities of autoimmune disease seemingly demonstrate deep problems in the natural preservation of an organic unity based on the overriding rule of self-tolerance. There are nonetheless those working in immunology who argue that Elrich's theory is misunderstood and that while selftolerance is evidently the rule, autoimmunity is always the exception. ${ }^{4}$ Moreover, it is further argued that rather than being a destructive incongruity of the immune system, the exception is always implicated in the maintenance of the rule. ${ }^{5}$ In other words, the selfdestructive anomaly is considered to play a productive role in the evolutionary survival of the organism.

Putting evolutionary determined equilibrium aside for now, the paradoxical relation between self and nonself in autoimmunity problematizes the assumed emergence of a 
natural state of immunity. The defences provided by the horror of self-toxicity will, on occasion, acquiesce, and the exception will overrule self-tolerance, possibly leading to the exceptional destruction of the organism. As follows, there is an ongoing debate concerning the value of the self/non-self metaphor as a theoretical tool to understand how cellular organisms defend against infections in immunology. For some, the metaphor's value plunged when it became evident that cells have the potential to be simultaneously anti-self and anti-foreign. Indeed, cellular behaviour seems to defy the terms set out by the immunological metaphor. As Robert S. Schwartz puts it, "the immune system, in short, does not operate by anthropomorphic principles such as "learn," "self," and "foreign," nor is there a sharp line between "self" and "foreign.",6

Taking this challenge to immunologic a step further, autoimmunity also questions how these same anthropomorphic principles are assumed to arrange social relations. In other words, similarly considered in terms of a broader concept, autoimmunity significantly complicates notions of communication, defence and regulation conventionally understood as immunologic processes. As follows, the concept prompts a novel approach that questions the ordering of social relations according to self/other relations. It tests the permeability of borders assumed to exist between self-identity and threats posed by an anomalous destructive nonself. As such, autoimmunity introduces a politics of exception, which unlike the negative binarisms of immunological exemption, can be grasped as an affirmative process.

The destructive and productive role of the viral anomaly in digital culture has been described as a topological spatiotemporal autotoxicity. ${ }^{7}$ This is 'a condition akin to a horror autotoxicus of the digital network', wherein the 'capacity of the network to propagate its own imperfections exceeds the metaphor with natural unity' ${ }^{8}$ Considered in stark contrast to immunologic, the topology of the digital network is the 'perfect medium' for spreading both 'perfection and imperfection' ${ }^{9}$ The goal now, it would seem (given that the virality of the digital network has become ever more interwoven with the social in so many profound ways), is to question how a more generalized concept of autoimmunity might be applied to social arrangements that occur when bodies and technologies are increasingly in concert with each other. Beyond digital contagion, it would seem, we encounter CTI. This is not just a digital infrastructure that confuses self and nonself, but mixtures of technologies and bodies that become socially arranged according to autotoxicity.

The notion that self/nonself relations are in a perpetual state of paradox is evidently nothing new. In order to grasp autoimmunity in this broader topological sense we do not need to look much further than Gabriel Tarde to resuscitate a social theory in which capricious contagious overspills are not regarded as anomalous, but become key to the arrangement of everyday social relations. ${ }^{10}$ Along these lines, relations do not simply become contagious; they are contagions. The arrangement of everyday relations established between humans and the world they inhabit are determined, as follows, by Tarde's imitative radiation. The CTI concept expands on this notion to ask what happens to the concept of self-identity when, as Tarde argued, the psychological sense of 'myself' is considered to be contaminated by the imitations of others. ${ }^{11}$ In other words, what 
happens when the self is understood to imitate the other to a point at which the relation between self and other - so important to both immunologic and anthropomorphic principles - collapses into a social cosmos of imitative relationality? Moreover, what occurs to social arrangements when the exception of self-toxicity is no longer considered an anomaly, but becomes the rule? Indeed, in a twenty first century social media world dominated by the contaminating sensory environments of market capitalism, it is arguably no longer self-harm that should concern us, but a tendency toward what Roger Caillois calls collective masochism. ${ }^{12}$ To establish this theoretical perspective I will again look to manifestations of digital culture; specifically, in this case, social media marketing, as a way to grasp how CTI can be used to rethink an age that is increasingly defined by radical self/nonself relations encountered in contagious social arrangements.

\section{Mirrors, Mimicry and Marketing}

Before exploring what the concept of CTI can achieve, it is necessary to trace the ways in which self/other relations have featured in academic marketing literature. To be begin with, it is important to note that a Tardean approach has made some impact on the study of marketing in terms of grasping, for example, the significance of pass-on-power, as well as co-production, affect and sentiment in consumption. ${ }^{13}$ But for the most part there is a marked tendency in more industry focused literature in particular to analyze the selfconcept in conventional ways that render it discrete in terms of how it interacts with others in sensory environments. At first glance, this discreteness is subtly conceived of. For instance, according to a recent academic study of adolescent photo sharing on social media the self and its interaction with others in the external world is considered 'integral to the creation and continuation of a stable harmonious self-concept' ${ }^{14}$ Adolescents in particular are assumed to upload and share photos of themselves adorned in various products as a way to crystalize the formation of their self-identity to present to others. ${ }^{15}$ However, this account of identity formation relies on a supposition that although selfidentity admits to social relations, the inner sense of self exists regardless of the external world. ${ }^{16}$ The notion that adolescents mimic others they encounter, by way of adorning similar cloths, is often downplayed, as such. In this kind of account the other functions mainly as a kind of collective mirror to help the adolescent grapple with the question: who am I? Group associations are important, but the self-concept is defined predominantly by representational internal choices made about these social associations, alongside apparently wilful consumption preferences for clothing, jargon, and music taste, for example.

This supposition is challenged by Tarde who insists that the imitations we find in these external relations should be the main focus of social theory. Indeed, Tarde countered individual and collective representations by presupposing "exactly what needs explaining, namely, "the similarity of millions of people." ${ }^{17}$ This is why, Deleuze and Guattari contend, "Tarde was interested instead in the world of detail, or of the infinitesimal: the little imitations, oppositions, and inventions constituting an entire realm of subrepresentative matter'. ${ }^{18}$ From a Tardean perspective there is indeed no need for a representational mirror to ensure that collective mimicry takes place. Arguably these mirroring processes present nothing more than a dream-of-action that supports an illusory 
boundary line between inner psychological experience of self and the outer world. To be sure, the aim of current social media marketing strategies is not, arguably, to infect the desire for a stable sense of the self, but rather leech the porosity of the psychological selfimage to the imitative radiations of the external world. It is this porosity to imitation that is readily exploited by marketers able to tap into big data assemblages providing a trace of (and adding a value to) what is imitated i.e. trending, shared, liked etc. That is to say, social media marketers do not infect the self, but rather harvest and activate a contagious social medium. It is this Tardean tendency toward social contamination and imitative radiation that marketers readily tap into (and encourage) in order to develop strategies of social influence.

Before expanding more on this tendency it is necessary to further grasp the role mirrors and mimicry have played in critical approaches to marketing that in many ways echo the immunologic. The inclination to lean on discrete concepts of self/other relations in recent marketing literature can certainly be traced back to much earlier attempts to decode the ideology of marketers working mainly in advertising in the 1960s and 70s. For instance, Judith Williamson's radical thesis on advertising introduces the Lacanian influenced concept of the 'Created Self'; arguing that the ideological intention of the marketer is to bring together groups of consumers, while at the same time individuating (and alienating) them. ${ }^{19}$ That is, creating a brand message that becomes an integrated part of a coherent sense of self to a point wherein the self becomes a commodity. Adverts that feature Pepsi People, for example, create an imaginary social medium, which offers the individual a Lacanian mirror-image of the external world to gaze into and aspire to. ${ }^{20}$ It is the aim of marketers to draw consumers into a desire to become one of the Pepsi People in order to feel good. But, evidently, such social aspirations and desires for perpetual happiness are delusional. This is how ideology is supposed to simultaneously provide a false image to mimic, while also individuating and alienating the sense of self. Indeed, Williamson sees Lacan's mirror as a metaphor for the external reflection of the self advertisers produce an ersatz imitation of sorts - encountered in the external world that estranges the self from the other. ${ }^{21}$ Nonetheless, despite its recognition of the external world, ideological advertising is significantly grasped like a mechanism of infection in which 'it is so crucial for the ad to enter you, and exist inside rather than outside your self-image: in fact, to create it'. ${ }^{22}$ Like the basis of Lacan's account of the mirror-image, the organism is, at first, so captivated by the external environment that it becomes its camouflage. In other words, mimicry is the capture of the organism by the environment rather than some evolutionary necessity to mimic surroundings in order to survive. But eventually this loss of self to the external world through captivation is transferred from the externality of the environment - bodily affects, camouflage etc. - to the inner world of phantasy - the narcissism of the ego and its false identification with the other. This is how, in short, bodily affects become phantasy in Lacan's mirror stage

To expand on this point we can see how differences and resemblances become important factors in determining an individuated self/other relation in the ideological analysis of advertising. Resemblances are located in the mirror images of people that appeal to you: people like you. Adolescent and adult consumers can, as such, compare themselves to the other, creating a sense of coherence that resonates with the brands they consume. But, at 
the same time, differences in ideological analysis are also found in the semiotic signification of the self/other relation. That is to say, the glimpse of the other (in the mirror-image of the ad) points to what you cannot be. Just as one cannot occupy the space of the mirror, one can never be happy all of the time. So despite the mimicry, the mirror-image makes consumers aware of what they are not. It makes them cognizant of their separation from the other and the environment they inhabit. ${ }^{23}$ Advertising is not only an infection of the inner world, it applies a kind of immunologic exemption to self/other relations insofar as the mirror-image isolates self from nonself and self from external world; producing an illusory, but stable sense of a narcissistic self-identity into which products and brands can be readily inserted. To be sure, Williamson regards the dream of a coherent self to be the object of the consumer's desire. Marketing consequently feeds on this desire for what is ultimately an unobtainable stability. This is the ideological creation of an infected self-concept, wherein the sense of self becomes a product in itself; the creation of a commodified self, no less.

There are some important variations in the ideological-semiotic analysis of advertising and Tarde's imitation thesis that need to be ironed out before we can fully explore autoimmunity. To begin with, although similar in some respects to Williamson's account, insofar as the object of desire is an illusory sense of self, what composes the dream of action in Tarde's society of imitation is not determined by a Lacanian mirror that gives rise to the representation of I. On the contrary, the object of Tardean desire is always belief. Again, there are subtle differences here that need to be considered. While beliefs may seem to offer a sense of inner stability, what is believed in is always imitated from the beliefs of others. The illusory sense of self acquired through mimicry is not therefore a representation of $\mathrm{I}$ in a mirror, but a contagious flow of imitated belief experienced at a sub-representational level. In other words, the sense of an inner coherence or selfidentity, which seems to separate the inner world from the external world, is the illusion. It is a dream of volition; a sense of self experienced in the reverie of social associations. The beliefs that are mimicked; that are imagined to belong to a self, belong to a social medium.

To further think through these differences in immunological orientated terms it is important to go back to the origins of Williamson's use of the Lacanian mirror. Lacan was famously inspired by Caillois's work on insects, fish, octopuses and mantises, and his significant challenge to the prevalent idea that mimicry is an instinctual form of protective immunity brought about by threats posed to the organism from the outside world. That is to say, the adaptable surface (or skin) of the animal becomes the first level of immunological defence. Rather than seeing these surface mimicries as protection for the organism against the external threat of the other, Caillois alternatively points to the many vulnerabilities that arise when the surface of an organism takes on the visual properties of its environment. The remains of mimetic insects are indeed as abundant in the stomachs of predators as those that cannot change their visual appearance. ${ }^{24}$ Caillois therefore begins to rethink the organism's mimicry of the environment not as a survival tactic, but as a capture of the subject in the spatial coordinates of its surroundings; an individual captured in a topology. As Parikka notes, 'Caillois addressed the function of mimicry not as a representation of figures or space but a spatial assemblage that bordered 
on disorder'. ${ }^{25}$ Mimicry is all about 'bodies in interaction' with the environment; that is, a relation between the haptic visual properties of space and affected bodies. ${ }^{26}$ Indeed, Parikka goes on to ponder a notion of affective relationality that in many ways counters the mirror stage insofar as it draws attention to the porous nature of the inside/outside relation to such an extent that the representational I is replaced by the space of the environment. ${ }^{27}$ The inner and outer world of phenomenological experience is therefore collapsed into an external world of affective relationality that disturbs the relation between personality and space. 'The reflective mind is forced to follow the noncognitive knowledge and motility of the body... [Caillois thus provides] a nonphenomenological mode of understanding the lived topology of the event'. ${ }^{28}$

Williamson's approach borrows from Lacan's reading of Caillois to argue that the outside image alienates the psychological self. However, there needs to be distinction made between the notion of advertising entering into, and occupying, the inner world of the consumer and a counter notion of a mimicry of bodily affects that challenges the selfconcept by locating social relations in a sensory environment. In other words, the ideological critique insists on the production of an inner personality (however delusional) while a nonphenomenological approach offers a theory of subjectivity produced by the capturing of individuals in space. The changes on the surface of the organism's body need not therefore become internalized in a reflective mind. As Caillois puts it, "the feeling of personality, considered as the organism's feeling of distinction from its surroundings, of the connection between consciousness and a particular point in space, cannot fail under these conditions to be seriously undermined'. ${ }^{29}$

There also needs to be a historical footnote added to this discussion which entertains a considerably older notion of infectious social relations and its increasing relevance to an understanding of social media marketing today. Indeed, again, there is nothing entirely new here. Although the apparent contagiousness of the nineteenth century crowds that inform Tarde's imitation thesis predate the mediated publics of the twentieth century, the objectives of twenty first century social media marketers seem to parasite the same externalized contagious tendencies he recognized. From this perspective, marketers do not need to infiltrate the self via the mirrors and mimicry of ideology, but instead they tap into the contagious social medium in which consumer beliefs about products and brands are readily passed on as affective contagions. As follows, marketing is not the creation of self-identity, but rather the production of sensory environments in which the contagions of a social medium can be encouraged. The social medium becomes the product. Social media is indeed an invention which appropriates desires always-already spreading through the external world.

\section{Social Media Marketing as Autoimmunity}

The potential exploitation of a contagious social medium can be readily observed in an experiment carried out by Facebook in 2014. This involved the manipulation of the emotional content of news feeds and measuring the effect these manipulations had on the emotions of 689,003 members of the social media phenomenon in terms of how contagious they became. ${ }^{30}$ The researchers who carried out the experiment found that 
when they reduced the positive expressions displayed by other users they produced less positive and more negative posts. Likewise, when negative expressions were reduced, the opposite pattern occurred. Although the recorded levels of contagion were rather paltry the researchers concluded that the 'emotions expressed by others on Facebook influence our own emotions, constituting experimental evidence for massive-scale contagion via social networks'. ${ }^{31}$ Indeed, even if this contentious and unethical attempt by Facebook to influence moods produced meagre evidence of contagion, the design and implementation of the experiment itself should alert us to a potentially Huxleyesque mode of mass manipulation. As Nicolas Carr contends, the bizarrely titled 'Experimental Evidence of Massive-Scale Emotional Contagion' through Social Networks draws attention to the way in which the cultivation of big data by marketers treats human subjects like lab rats while also pointing to the widespread nature of manipulation by social media companies. 'What was most worrisome about the study', Carr contends, 'lay not in its design or its findings, but in its ordinariness'. ${ }^{32}$ This kind of research is indeed part of a 'visible tip of an enormous and otherwise well-concealed iceberg' in the social media industry. ${ }^{33}$ To be sure, the one thing that both the disparagers and apologists for social media seem to agree on is that user manipulation is rife on the internet. It is, after all, what every social media business enterprise strives to do.

Social media networks are the perfect medium for sharing and, it would seem, a test bed, or nursery, for cultivating and igniting emotional contagions. Unlike broadcast media, which similarly spread emotions, the users of these networks are predisposed, it would seem, to routinely share their feelings in exchange for the tools that allow them to freely do so without concern for how these tools might, in turn, be used to manipulate them. Of course, despite the relatively small scale media storm of outrage surrounding this particular attempt to manipulate emotions, many Facebook users will be oblivious to their participation in this research, or indeed, their assumed inclination to respond to emotional suggestion in such an apparently porous and imitative fashion. Moreover, most Facebook users will be unaware of the role their ignited emotional responses play in an infectious social medium; oblivious to, that is, the way in which emotions are being harvested in the data assemblages mined by corporate social media.

Beyond these ethical concerns, Facebook's emotional contagion research can also be grasped as part of a trajectory of digital autoimmunity. Indeed, ever since the invention of the online marketplace, marketers have arguably striven to emulate the infectiousness of biological and computer viruses so as to surreptitiously spread marketing messages through social networks unawares. These efforts can be seen as a part of two waves of viral marketing in which marketers have increasingly lent on the idea that an infected host will pass on a message more effectively through its own networks than it would through conventional media channels. Although the ignition of virality is never certain, in the first wave, the low cost of viral marketing was a good enough incentive, it would seem, for business enterprises to switch from expensive broadcast media to viral networks. Moreover, the prospect of evading the conscious tyranny of mass marketing by simultaneously secreting away messages in everyday communications and turning consumers into the medium for potential contagions also offered novel unconscious neural pathways for marketers to spread their messages. 
The Facebook research on emotional contagion represents a considerable development on the immunological and memetic theoretical models that underpinned the first wave of cost effective viral marketing. Indeed, whereas the template for the first wave was based on a configuration of virulent memes and immunological disease thresholds the second wave seems to sidestep the defensive horror of autotoxicus by looking to collapse the immunological distinctions between self, nonself and the social medium in which they become assembled. Perhaps this shift from a first to a second wave of viral marketing can be best observed in recent challenges to the immunologic model posed from within marketing itself. ${ }^{34}$ This is a challenge to the notion that the trigger for contagious overspills can be found in network clusters that develop around influential and promiscuous individuals. Here Malcolm Gladwell's crude version of threshold theory is used to explain the point at which a small trend tips over into a big contagion. That is, wherein the number of infected nodes outlives the number of uninfected nodes. A mainstay of viral marketing, Gladwell's Tipping Point thesis is nevertheless confronted by Duncan Watts's contention that contagions are the product of the 'infectability of the networked world' ${ }^{35}$ This is a point of departure that has not been overlooked by social media marketers. The research focus has in fact significantly shifted away from influential nodes toward the design and production of infectable small world networks that prime mood and ignite capricious affective contagions. ${ }^{36}$

Despite initial claims to have captured what gets passed on in a social network in practical terms, first wave viral marketing was still pushing messages out to an online audience paying attention to websites in much the same way as they would mass media. Designers were engaged in producing sticky experiences that encouraged the cultural practice of passing on content. The big difference with social media today is that it rouses the passing on of viral content through such tactics as following, liking, sharing etc., ensuring that complex and capricious contagions are more readily mined and prompted into action. In current big data research there is a continuing focus on producing small changes intended to lead to large scale contagions. For example, in the work of Watts we see how exceptional contagions are, and how, as such, the affective priming and manipulation of the mood of the social medium becomes the main focus. It is not therefore the infected subject that matters, but rather the capricious encounters in affective atmospheres that might ignite contagion.

\section{Autoimmunity as Collective Masochism}

To add a Tardean slant to these developments, we might say that the second wave of viral marketing denotes the dissolution of the infected subject into CTI. The infectious mimicry of autoimmunity is, as such, no longer (borrowing from immunology again) about self-harm; it is perhaps, following Caillois, all about collective masochism: phenomenon he located in the sensory environments of mimicking animals.

[T] here are cases in which mimicry causes the creature to go from bad to worse: geometer-moth caterpillars simulate shoots of shrubbery so well that gardeners cut them with their pruning shears. The case of the Phyllia is even 
sadder: they browse among themselves, taking each other for real leaves, in such a way that one might accept the idea of a sort of collective masochism leading to mutual homophagy, the simulation of the leaf being a provocation to cannibalism in this kind of totem feast. ${ }^{37}$

The point is that as the market colonizes and absorbs the sensory environment in which human mimicry takes place, these molecular imitations will inevitably become more oriented toward mimicking products and brands. In other words, mimicry is an outcome of the capture of subjectivity by the space of the market. Significantly, this is not a subject that can freely identify with an I within space, since it is the space itself that positions the subject in the market. Unlike Williamson's focus on commodification of the self, which occurs inside the subject, we need to look to the topological relations that capture subjectivity. This point can be expanded upon by returning to the adolescent photo-sharing experiences discussed above. We can see here how young consumers, adorned in the products and brands they find in the shopping malls, spread their mimicry of the sensory environment via social media to CTI that prompt more consumers to become coordinates in the topological grid of the market. In classic Tardean terms then, this is imitative radiation; it is a molecular mimicry that helps to explain the similarity of millions of people. As Caillois similarly puts it, we find a kind of magic attraction in these topologies in which 'like produces like'. ${ }^{38}$

In the first wave of viral marketing the infected body becomes the marketing message. It is, in itself, the virus that passes on the message. The infected body becomes the host medium of contagion. In the second wave the relation between self and nonself has not only become more blurred in a topological sense; it has also been captured by the space of the market. It is not the body, but the social medium that is now the virus. Indeed, by way of occupying the sensory environments in which relations are made, the market has laid down the spatial and temporal conditions of social arrangements. It influences, in this way, what gets imitated and what gets passed on. Williamson's response to this capture of subjectivity by the space of the market is to try to decode the infected self; that is to say, grasp the creation of the self by the market. The intention is, it seems, to provide the individual with the semiotic tools with which to see through the ideological trickery of advertising. However, when consumers become the medium of infection the sense of an inside being infiltrated by an outside becomes lost in the spatial capture of a topology.

There is no incoming message to decode. Indeed, consumers are no longer able to decode marketing messages since they have become participants in an infectable space in which they co-produce and pass on code. The second wave is profound in this sense, since it is no longer the market that directly infects, but consumer collectives that indirectly infect others in their neighbour. Facebook's emotional research creates, as such, a topology, not a self, in which looping affective relations, ignited by online content (newsfeeds), spread through pass-on power. The big data lab rat unconsciously becomes part of a mischief of rats oblivious to the viruses they carry and seemingly insensible to the prospect of becoming an active carrier for brand messages.

\section{Autoimmunity, Anxiety and Transindividuality}


Becoming viral is still a practice embraced in marketing circles, which builds on the popular discourses of gurus like Gladwell and Watts and is sold on as a commodity in the form of the best seller, the business seminar and Ted Talk. But a second wave of viral marketing needs to be grasped as part of a more general and shifting immunological discursive formation, which at first territorialized populations around the visceral fear of the nonself, but now ignites a wider range of contagions including many joyful encounters with affective capitalism. Yet, despite this reorientation, immunologic anxiety has not entirely disappeared from view. The blissfully unaware Facebook lab rats might one day wake up from their somnambulistic stupor. The anxiety experienced will not be the outcome of a realization that the sense of self has been occupied by the market because, as we have seen, this is not the nature of the spatial capture of subjectivity. The anxiety felt will, in contrast, be experienced through the terrifying apprehension that the space occupied (and mimicked) is a dream of action. Indeed, as is argued below, it is the conditions of CTI in the social medium itself that causes new anxieties to unfold. Social media activists and researchers alike need to therefore better understand the dynamics of this topological spatial capture.

The relational arrangements between individuals and the topological space they occupy requires a radical rethink. Developing on Parikka's reading of Gilbert Simondon, we might begin by considering CTI not as a spatial backdrop in which communication takes place, but rather as 'an active milieu of relations'. ${ }^{39}$ This is a topological space that mediatizes the individual; producing intensive individuation processes that 'situate the subject in the world' ${ }^{40}$ In the novel social arrangements of this space, the prediscursive anxieties relating to contagion experienced in the immunologic model are substituted by new anxieties concerning the extent to which already infected bodies becomes part of a seemingly inescapable contagious social medium. That is, a sudden realization that what was regarded as a sense of ownership over the psychological self, which seemed to belong to the $\mathrm{I}$, is in fact part of a shared data assemblage steered by the market researchers at Facebook. The trending posts we saw that made us laugh, get angry and cry; the posts we passed on to our friends and followers as shares and likes. They were mostly ersatz experiences. This is a moment of anxiety when the individual becomes aware that they are part of a collective virus and actively engaged in passing it on by way of their interactions with others. These are moments of anxiety when individuals become 'aware of their presence as part of the 'informational collective' that shapes online activity' and emotional experiences. ${ }^{41}$ This is perhaps akin to a Simondonian anxiety wherein the difference between individuality and collectivity collapses into an affective state of transindividuality. ${ }^{42}$

\section{Immunity to Relational Media}

Back in 2006, the information architect and design consultant, Adam Greenfield, drew our attention to a near future digital culture in which ubiquitous computing makes everyday life 'fiercely relational'. ${ }^{43}$ Pervasive computing introduces a lived relationality that is not simply experienced when individuals become a set of values stored in a database. This is already a reality we find in our experiences with social media. But it will also be increasingly realized in the way in which such values, including spatial 
proximity to points of consumption, location traces and emotional dispositions, are matched against the values belonging to others. As computing becomes ever more ubiquitous these relational values will exert a 'transformative influence' on social relations. ${ }^{44}$ Indeed, if we are to follow, as Greenfield does, Erving Goffman's notion that we are all actors wearing a collection of masks we switch between in order to manage self-identities exposed to ever changing social situations, then we need to grasp how relational media threatens to make the sustaining of different masks 'untenable' ${ }^{45}$ In the age of social media, the personal in computing has certainly moved into the social domain where the private and public performances of Goffman's masked actors collapse into data-bodies assembled by relational databases.

Beyond Greenfield's account, however, we might also venture that relational media draws attention to a much older problem than who controls the 'custody of selfconsciousness. ${ }^{46}$ That is; the problem of what happens to individual and collective experiences when exposed to all-pervasive technology. This problem clearly maps onto the shift in immunological orientation highlighted in this article. Indeed, given the proclivity of social media, and pervasive computing, toward autotoxicity, it is the relational aspects of immunology, particularly with regard to the sensory relations established between humans and technology, which require attention. It is therefore with Ellis and Tucker's recent social psychology reading of Simondon's technics that I now conclude with, since as they appropriately claim, not enough work had been done on this relation. ${ }^{47}$ To begin with, the point needs to be made that although social media adapts the way in which a person experiences individuating events, following Simondon, the focus on the individual must not be confused with the constituted being. Thus as personal life increasingly shifts from individuals to the huge databases of governments and commercial organizations, the intersection between humans and technology unfolds as part of processes of individuation, which do not have to be human. ${ }^{48}$ As Ellis and Tucker note, Simondon does not ontologically, as such, separate bodies and technology because both are part of the experience of individuation. ${ }^{49}$ Indeed, human and technological individuations are the threshold point at which human dreams and machines become mixed. However, this is not to say that a nightmarish tension or deep anxiety does not exist in this coming together of bodily and technological arrangements. As they assemble, something novel and unknown emerges.

As is the case with immunology, the psychic self becomes a conceptual problem that cannot be defined by internal properties alone. It is rather an affective relation, produced through processes, not separate categories. Similarly, like Tarde's imitative subjectivity, an individual 'always-already carries some of the collective with it' ${ }^{50}$ The resulting anxiety is not therefore equivalent to the alienation established between immunological self and nonself, or is it, for that matter, akin to Williamson's alienating mirror image. On the contrary, this is anxiety felt as an outcome of the 'coming together' of technologies and bodies, forming new collective arrangements and experiences that challenge immunological, and potentially anthropomorphic, divisions. This is not a tension-free experience since the individual psyche always-already experiences collective baggage as an anxiety. In autoimmunity terms this is perhaps a new kind of horror that emerges through an experience that is both individual and collective - self and nonself - at the 
same time. That is what Simondon refers to as the preindividual. In other words, anxiety arises through an unresolvable perceptual problem of individuation and preindividuation. This occurs because of 'the reality that the individual experiences itself as a unique subject, but at the same time recognises itself as partially collective' ${ }^{51}$ Returning to the example of the Facebook lab rat, it is the revelatory experience of realising that our emotional experiences are not ours that produces a new horror.

How then to overcome the anxieties ignited in the experiences of CTI? One consideration might be to continue to follow Simondon's solution to anxiety in the concept of the transindividual. This is not a reconstitution of individual or collective categories. As Ellis and Tucker point out, anxiety cannot be resolved inside the subject or the collective. ${ }^{52}$ It requires a perceptual bridge that might enable a softer passage through the individual-collective continuum. This is a bridge that counters internal and external spaces, working across boundaries of self and nonself. Moreover, the transindividual escapes spatial coordinates - it is not a grid, it is a becoming, which may indeed frustrate the colonising spaces of market orientated mimicry. It might, as such, provide the basis of a relational immune system (RIS) that does not look to perceive of something that exists outside the self as a nonself, but perceives of a 'multiplicity of perceptual worlds' ${ }^{53}$ It is this notion of a RIS that needs more work. Indeed, in contrast to the binary between the phenomenological I and the crowd brains of mass psychology there needs to be an investigation into these relational bridges that span across multiple perceptual worlds. This might lead to a critical social psychology of the transindividual which invests in care systems designed to lessen the anxiety of transindividuation as well as defending against the horror of marketing contagions intended to surreptitiously piggyback the imitative nature of this relation.

Notes

${ }^{1}$ Jussi Parikka \& Tony D. Sampson, The Spam Book: On Viruses, Porn and Other Anomalies From the Dark Side of Digital Culture (New Jersey: Hampton, 2009), 15 and Tony D. Sampson, Virality: Contagion Theory in the Age of Networks (Minneapolis: Minnesota University Press, 2012), 127-143.

${ }^{2}$ Ganaele Langlois and Greg Elmer, 'The Research Politics of Social Media Platforms' Culture Machine Vol 142013 Open Humanities Press.

http://www.culturemachine.net/index.php/cm/article/viewArticle/505

${ }^{3}$ Ibid., 5.

${ }^{4}$ Zoltan A. Nagy, A History of Modern Immunology: The Path Toward Understanding (New York: Elsevier, 2014), 241.

${ }^{5}$ Ibid.

${ }^{6}$ Robert S. Schwartz, 'Review of The Immune Self: Theory or Metaphor?' New England Journal of Medicine (332) 1995, 1176-77 http://www.nejm.org/doi/full/10.1056/NEJM199504273321718 (accessed $24^{\text {th }}$ August 2015).

${ }^{7}$ Parikka and Sampson, The Spam Book.

${ }^{8}$ Ibid., 15.

${ }^{9}$ Ibid. 
${ }^{10}$ Gabriel Tarde, The Laws of Imitation, trans. E. C. Parsons (New York: Henry Holt, 1903).

${ }^{11}$ Gabriel Tarde, Penal Philosophy (New Jersey: Transactional Publisher, 2001), 116 -

118. Originally published in 1921.

12 Roger Caillois, 'Mimicry and Legendary Psychasthenia' (1936) trans. John Shepley in October 31 (1984), 24-25.

${ }^{13}$ Nigel Thrift, 'Pass It On: Towards a Political Economy of Propensity' in Matei Candea, ed., The Social after Tarde: Debates and Assessments (London: Routledge, 2010), 248-70 and Adam Arvidsson and Giannino Malossi, 'Customer Co-Production from Social Factory to Brand: Learning from Italian Fashion' in Detlev Zwic and Julien Cayla (eds.), Inside Marketing: Practices, Ideologies, Devices in (Oxford: Oxford University Press, 2011), 216-18.

${ }^{14}$ Jenna Drenten, 'Snapshots of the self: exploring the role of online mobile photo sharing in identity development among adolescent girls' in Angeline G. Close (ed.), Online consumer behavior: theory and research in social media, advertising, and e-tail (New York: Routledge, 2012), 15.

${ }^{15}$ Ibid., 6.

${ }^{16}$ Ibid.

${ }^{17}$ Gilles Deleuze and Félix Guattari, Anti-Oedipus (London: Athlone Press, 1984), 21819.

18 Ibid.

${ }^{19}$ Judith Williamson, Decoding Advertisements: Ideology and Meaning in Advertising (London: Marion Boyars, 1978).

${ }^{20}$ Ibid., 63-64.

${ }^{21}$ Ibid., 63.

${ }^{22}$ Ibid., 48.

23 Ibid., 63.

${ }^{24}$ Caillois, 24-25.

25 Jussi Parikka, Insect Media: An Archaeology of Animals and Technology (Minneapolis, University of Minnesota Press, 2010), 97.

26 Ibid., 98.

${ }^{27}$ Ibid., 104.

${ }^{28}$ Ibid., 100.

${ }^{29}$ Caillois, 28.

${ }^{30}$ Adam D.I. Kramer, et al., 'Experimental evidence of massive-scale emotional contagion through social networks' Proc Natl Acad Sci USA, 111(24) (2014), 8788-8790. http://www.pnas.org/content/111/24/8788.full

${ }^{31}$ Nicholas Carr, 'The Manipulators: Facebook's Social Engineering Project' The Los Angeles Review of Books (September 14th, 2014).

$\mathrm{http} / / /$ lareviewofbooks.org/essay/manipulators-facebooks-social-engineering-project\#

32 Ibid.

33 Ibid. 
34 Tony D. Sampson and Jussi Parikka 'Learning from Network Dysfunctionality: Accidents, Enterprise, and Small Worlds of Infection' in John Hartley, Jean Burgess, Axel Bruns (eds.), A Companion to New Media Dynamics (Chichester: John Wiley, 2013), 450-460.

35 Ibid., 457.

36 Ibid.

${ }^{37}$ Caillois, 25.

${ }^{38}$ Ibid.

${ }^{39}$ Parikka, Insect Media, 140-44.

40 Ibid., 141. Simondon cited in.

${ }^{41}$ Darren Ellis and Ian Tucker, The Social Psychology of Emotion (London: Sage, 2015), 178.

42 Ibid., pp. 171-179.

${ }^{43}$ Adam Greenfield, Everyware: The Dawning Age of Ubiquitous Computing (New York: New Riders, 2006), 81.

${ }^{44}$ Ibid., 84.

${ }^{45}$ Ibid., 86-87.

${ }^{46}$ Ibid., 86.

${ }^{47}$ Ellis and Tucker, 171.

48 Ibid., 172.

${ }^{49}$ Ibid., 173.

${ }^{50}$ Ibid., 174.

${ }^{51}$ Ibid., 175.

52 Ibid.

${ }^{53}$ Ibid., 174 